\title{
ESTIMATION DE LA BIOMASSE AÉRIENNE À PARTIR DE DONNÉES LIDAR AÉROPORTÉ
}

\author{
António Ferraz ${ }^{1,2}$, Clément Mallet $^{1}$, Stéphane Jacquemoud ${ }^{3}$, Gil Gonçalves ${ }^{2,4}$, Paula Soares $^{5}$, Luisa Pereira ${ }^{6}$, \\ Margarida Tomé 5
}

1 : IGN, MATIS, 73 avenue de Paris, 94160 Saint Mandé, France ; Université Paris-Est

2 : R\&D Unit INESC Coimbra, R. Antero de Quental 199, 3000-033 Coimbra, Portugal

3 : Institut de Physique du Globe de Paris - Sorbonne Paris Cité, Université Paris Diderot UMR CNRS 7154, Case 7011, 35 rue Hélène Brion, 75013 Paris, France

4 : Departamento de Matemática, Universidade de Coimbra, Apartado 3008, 3001-454 Coimbra, Portugal

5 : Universidade Técnica de Lisboa, Instituto Superior de Agronomia

Centro de Estudos Florestais, Tapada da Ajuda, 1349-017 Lisboa, Portugal

6 : Universidade de Aveiro, Escola Superior de Tecnologia e Gestão de Águeda, apartado 473, 3754-909 Águeda, Portugal

\begin{abstract}
Résumé
Dans le cadre des politiques de lutte contre le réchauffement climatique (programme UN-REDD des Nations Unies), les techniques de télédétection sont désormais utilisées pour extrapoler des estimations de la biomasse aérienne à l'échelle locale (dérivées à partir des mesures terrain obtenues via des inventaires forestiers) sur des grandes surfaces où aucune donnée de référence n'est disponible. Néanmoins, la communauté scientifique signale encore une grande incertitude concernant la quantification du $\mathrm{CO}_{2}$ stocké dans les forêts. Cela est dû au nombre insuffisant d'échantillons terrains sur des grandes zones de forêt de la planète, notamment dans des pays qui n'ont pas de pratiques régulières d'inventaires forestiers. Dans cet article, nous investiguons la fiabilité de la technologie lidar aéroporté (airborne laser scanning, ALS) pour estimer la biomasse aérienne des forêts sans nécessiter d'importantes mesures terrain. D'abord, des métriques forestières clés qui sont couramment obtenues via les inventaires forestiers traditionnels sont dérivées par analyse des donnés lidar. II s'agit notamment de la densité d'arbres, leur hauteur individuelle, l'épaisseur de la couronne, la hauteur moyenne de chaque strate, et le taux de couverture de canopée de chacune de celles-ci. Puis, des équations allométriques sont utilisées pour estimer la biomasse à partir des mesures terrain ainsi que des mesures lidar. Enfin, les résultats sont évalués pour chaque strate de végétation sur 40 parcelles d'une forêt portugaise à plusieurs strates de végétation. La biomasse aérienne est dérivée à l'échelle de la placette forestière avec une erreur de $0,4 \%$ comparée aux estimations terrain, ce qui prouve que notre approche est un outil efficace pour estimer la biomasse aérienne avec précision. Le développement de techniques similaires fondées sur le lidar aéroporté, capables de traiter forêts de différents biomes, avec l'utilisation conjointe de mesures obtenues par télédétection satellitaire, serait une avancée significative pour l'estimation de la biomasse sur de vastes régions à l'échelle mondiale.
\end{abstract}

Mots clés : Lidar aéroporté, biomasse aérienne, forêt multi-couche.

\begin{abstract}
In the framework of climate mitigation policies through UN-REDD, remote sensing techniques have been commonly used to coherently extrapolate local reference aboveground biomass (derived from forest metrics obtained by means of traditional forest inventories) over large areas over which there is no field measurements available. However, the scientific community still reports a large uncertainty about the amount of $\mathrm{CO}_{2}$ stored in forests. This is due to the lack of field samplings over large areas around the world, namely in countries without systematic forest inventories practices. In this work, we investigate the reliability of the airborne laser scanning (ALS) technology in order to estimate biomass without the need for massive field measurements. First, forest metrics, commonly acquired by means of field inventories, are derived from ALS data, namely tree density, individual tree height, crown size, single strata mean height, and single strata canopy cover. Secondly, allometric equations tailored for aboveground biomass estimations are fed by either field-and ALS-derived forest metrics. Finally, results are assessed for single strata (overstory, understory and ground vegetation) over 40 stands of a multi-layered Portuguese forest. Forest stand level aboveground biomass is derived with an error of $0.4 \%$ of field estimates that proves that our approach is a reliable tool to derive accurate aboveground biomass accurately. The development of similar ALS-based techniques able to deal with different forest biomes used in combination with satellite remote sensing measurements would be a significant advance for aboveground biomass estimation over extensive regions all over the world.
\end{abstract}

Keywords : Airborne laser scanning, above-ground biomass, multi-strata biomass. 


\section{Introduction}

La mesure et le suivi de la biomasse aérienne (aboveground biomass - AGB) de la végétation sont devenus ces dernières années un sujet de recherche de premier ordre. Cela est dû à son rôle très important dans le cycle du carbone mais également dans les négociations internationales sur le climat (Zolkos et al., 2013). En 2009, la Convention Cadre sur le Changement Climatique des Nations Unies (United Nations Framework Convention on Climate Change - UNFCCC) a établi les instructions pour l'évaluation des stocks de carbone dans les forêts, à l'échelle mondiale, via l'implémentation du programme UN-REDD (Reducing Emissions from Deforestation and Forest Degradation (Houghton et al., 2010)). Ces trois composantes sont: (i) la mesure de l'étendue spatiale des forêts et de leurs changements; (ii) l'évaluation des stocks de carbone et des émissions; (iii) la vérification des constatations et de la mise en pratique des activités UN-REDD.

Les techniques de télédétection sont adaptées pour la mesure et le suivi des forêts sur de grandes étendues spatiales. Par conséquent, ce sont des outils cruciaux pour satisfaire aux trois exigences de I'UNFCCC (Hall et al., 2011). Toutefois, ces techniques ne permettent pas une mesure directe de la biomasse aérienne. En fait, les mesures forestières obtenues nécessitent une étape de calibration (menée souvent à travers des modèles de régression linéaire) en utilisant des donnés d'AGB de référence, dérivées à partir de mesures terrain (Wulder et al., 2012). Une fois que ces mesures ont été correctement calibrées, des cartes de biomasse aérienne sont alors calculées sur les zones couvertes par les données de télédétection. Le succès de cette approche dépend fortement à la fois de la quantité et de la qualité de la biomasse estimée à partir des données terrain : un nombre non négligeable de mesures doit être collecté afin de bien restituer la variabilité spatiale de la biomasse aérienne pour une forêt donnée (Saatchi et al., 2011). Ainsi, une estimation fiable de la séquestration du carbone à l'échelle mondiale nécessiterait plusieurs systèmes d'inventaire forestier à des niveaux locaux et régionaux, pour une grande variété de sites géographiques, avec de fréquentes revisites et avec une stratégie haute-densité d'échantillonnage spatial (Gobakken et al., 2012). En effet, le coût financier trop important des inventaires forestiers terrain rend impossible leur seule utilisation pour établir un réseau robuste de données de référence sur la biomasse aérienne (Gonzalez et al., 2010).

Dans cet article, nous étudions la pertinence du lidar aéroporté pour réduire l'énorme besoin en mesures in situ pour l'estimation de la biomasse aérienne. Un lidar aéroporté fournit des nuages de points en trois dimensions permettant une modélisation fiable, peu bruitée et de très haute résolution spatiale de la structure des zones forestières. Cette technologie permet l'obtention directe d'un grand nombre de métriques forestières qui sont habituellement mesurées par des campagnes terrain pour alimenter les équations allométriques de biomasse : il s'agit de la hauteur de chaque arbre, l'épaisseur de la couronne, la hauteur moyenne de chaque strate et le taux de couverture verticale (vertical canopy cover, $v c c$ ) par strate. Une telle approche permet d'éviter l'étape de calibration nécessaire à l'estimation de telles métriques (Hyyppä et al., 2008). Bien que les méthodes fondées sur l'analyse de données lidar aéroporté ont été validées pour de nombreuses métriques telles que la densité d'arbres, la hauteur individuelle des arbres (Morsdorf et al., 2004 ; Popescu et Wynne, 2004 ; Solberg et al., 2006 ; Reitberger et al., 2009; Hu et al., 2014) ou le $v c c$ (Korhonen et al., 2011; Wing et al., 2012), leur fiabilité pour l'estimation de la biomasse n'a été que peu abordée dans la littérature (Popescu, 2007). Ici, des métriques obtenues par mesures terrain et par analyse des données lidar sont intégrées à un ensemble d'équations allométriques de biomasse et les résultats obtenus sont comparés. Des méthodes développées auparavant pour l'extraction d'arbres individuels et de la hauteur moyenne des strates de végétation (Ferraz et al., 2012) ainsi que pour l'estimation du vcc (Ferraz et al., soumis) permettent d'évaluer la biomasse aérienne à une échelle identique à celles de l'approche fondée sur les données d'un inventaire terrain, i.e., à l'échelle de l'arbre pour la canopée, et à l'échelle de la strate pour le sous-bois et la végétation arbustive. Des résultats sont présentés sur 40 placettes forestières d'une forêt portugaise multi-strates.

\section{Méthodologie}

Cette section est divisée en trois grandes parties. Tout d'abord, les mesures terrain ainsi que les données lidar aéroporté acquises sur notre zone d'intérêt sont décrites (Section 2.1). Ensuite, la biomasse est estimée à partir d'un ensemble de mesures terrain via un ensemble d'équations allométriques (Section 2.2). Dans la section 2.3.1, nous détaillons alors brièvement les méthodes employées pour dériver l'ensemble de métriques forestières par analyse de données lidar aéroportées. Finalement, la biomasse mesurée à partir des données lidar est évaluée par comparaison avec le même jeu d'équations allométriques (Section 2.3.2).

\subsection{Données}

La zone d'étude se situe près de la ville d'Águeda au nord-ouest du Portugal $\left(40^{\circ} 36^{\prime} \mathrm{N}, 8^{\circ} 25^{\prime} \mathrm{W}\right)$. Elle couvre près de $9 \mathrm{~km}^{2}$, son altitude varie de 70 à $220 \mathrm{~m}$, et les pentes vont de 2,5 à 34,2\%. Le site est composé de manière prédominante d'eucalyptus (Eucalyptus globulus Labill.), avec quelques parcelles de pins maritimes (Pinus pinaster Ait.), de buissons et de champs. La canopée est plutôt éparse et le sous-bois est assez bien développé. La végétation arbustive est plutôt luxuriante, ce qui est typique des forêts méditerranéennes. La strate 
de sous-bois est principalement composée d'arbres opprimés (eucalyptus, pin maritime, acacia et chêne), d'ajoncs (Ulex spp.), de landes (Erica spp.), de genêts (Pterospartum spp.), de cistes (Cistus spp.), de mûres (Rubus spp.), de fougères et de plantes herbacées.

\subsubsection{Mesures terrain}

47 placettes ont été identifiées puis inventoriées en suivant un protocole terrain mis au point par l'Inventaire Forestier National Portugais (AFN, 2009). Toutefois, trois placettes avec de la broussaille impénétrable ont été éliminées tout comme deux autres couvertes de pin maritime n'ont pas été prises en compte dans cette étude car nous ne disposions pas des équations allométriques pour l'estimation de la biomasse adéquates pour le territoire portugais. Enfin, deux placettes de moins d'un an d'âge ont été de même laissées de côté car elles n'étaient pas très significatives en terme de biomasse. En conséquence, seules 40 placettes composées d'eucalyptus ont été mesurées. Chacune est composée de deux cercles concentriques, un extérieur $\left(400 \mathrm{~m}^{2}\right)$ et un intérieur $\left(200 \mathrm{~m}^{2}\right)$, délimitées au décamètre. La forêt étant très morcelée et appartenant à un grand nombre de propriétaires, il se peut qu'une placette contienne plus qu'un peuplement forestier, i.e., qu'elle ne soit pas uniforme en terme d'espèces d'arbres et de plantes, d'âge et d'arrangement spatial (Stokes et al., 1989). Dans de telles configurations, seul le peuplement situé au centre de la placette a été inventorié. En conséquence, les mesures ne concernent qu'un seul peuplement forestier à l'intérieur des cercles concentriques : cela signifie en pratique que les zones cartographiques sont de taille variable. L'âge des parcelles va de 1 à 13 ans. Cellesci ont été segmentées en deux groupes correspondant d'une part à 10 parcelles juvéniles (1-4 ans), et d'autre part à 30 parcelles adultes (> 4 ans). Les parcelles juvéniles sont composées soit de deux strates (végétation arbustive et canopée), soit de végétation arbustive seule. Les parcelles adultes comprennent elles trois couches de végétation (végétation arbustive, sous-bois et canopée), même si parfois, certaines parcelles peuvent ne pas avoir de sous-bois. Cela est dû à des activités d'élagage.

Le protocole terrain spécifie que pour chaque arbre de plus de $2 \mathrm{~m}$ de hauteur il est nécessaire de renseigner son espèce, son diamètre à hauteur de poitrine $(d b h$, $\mathrm{cm})$, sa hauteur $(t h, \mathrm{~m})$ et la hauteur de la base de sa couronne $(c b h, \mathrm{~m})$. Le diamètre à hauteur de poitrine a été mesuré à l'aide d'un compas alors que th et $c b h$ l'ont été avec une canne téléscopique ou avec un Vertex. Finalement, les arbres ont été individuellement classés selon leur statut social : arbre dominant, co-dominant, dominé ou opprimé. En ce qui concerne le sous-bois et la végétation arbustive, il apparaît extrêmement fastidieux d'évaluer ainsi toutes ces métriques forestières pour chacune des plantes. Ces couches sont alors en pratique caractérisées à l'échelle de la placette en utilisant la hauteur moyenne $(\bar{h}, \mathrm{~m})$, le taux de couverture verticale $(v c c, \%)$ et la prédominance $(\%)$, qui est en effet le $v c c$ échantillonné par espèce. Le protocole terrain spécifie que deux opérateurs distincts doivent estimer visuellement et séparément ces métriques. Quand leurs estimations sont similaires, la valeur moyenne est conservée. Sinon, ils cherchent à atteindre un consensus. Notons enfin que les mesures aux échelles de l'arbre et de la parcelle ont été obtenues à la fois dans les cercles extérieur et intérieur.

Les informations collectées lors d'inventaires forestiers sont souvent de précision géométrique inférieure à celles des systèmes lidar aéroportés. Afin de les améliorer, un réseau géodésique local composé de 41 paires de points obtenus par mesures GPS a été établi dans la même projection cartographique que les données lidar aéroporté (Gonçalves et Pereira, 2012). Tous les centres de parcelles et l'intégralité des positions des arbres situés à l'intérieur du cercle extérieur ont été relevés avec un tachéomètre. Enfin, toutes les données ont été intégrées dans la même géométrie 3D.

\subsubsection{Données lidar aéroporté}

Les données lidar aéroporté ont été acquises le 14 juillet 2008 avec un système LiteMapper 5600 en mode retour d'onde complète (Hug et al., 2004). La tâche au sol était de $0,3 \mathrm{~m}$ et l'angle d'acquisition couvrait $\pm 22,5^{\circ}$. Un recouvrement entre bandes de $70 \%$ a été choisi afin de garantir une couverture continue et redondante du terrain. La densité moyenne de points par bande est de $3,3 \mathrm{pts} / \mathrm{m}^{2}$ et est de $9,5 \mathrm{pts} / \mathrm{m}^{2}$ en moyenne pour les placettes acquises une fois que toutes les bandes lidar ont été ajustées (valeurs minimale $=4,7$, maximale $=15,5$ et $\sigma=1,9 \mathrm{pts} / \mathrm{m}^{2}$ ). Les ondes lidar numérisées ont été traitées afin de générer un nuage de points 3D (outil RiANALYZE) : chaque onde enregistrée est la source de 1 à 5 échos donc d'autant de points 3D. Les mesures du système hybrique GPS/INS de géoréférencement direct ont permis d'obtenir la position et l'orientation de la plateforme avec une forte fréquence temporelle, qui ont été ensuite corrigées par ajustement des bandes lidar adjacentes. Ces données, couplées à une station GPS fixe, permettent d'obtenir un nuage de points 3D exprimé dans le système de coordonnées WGS84/UTM zone $29 \mathrm{~N}$. Enfin, les erreurs systématiques d'altitude ont été éliminées en utilisant des données de contrôle qui sont ensuite propagées à l'intégralité de la zone acquise. Afin d'obtenir la hauteur exacte des objets de la zone, les points 3D ont été filtrés en points sol et végétation. Une triangulation de Delaunay a été calculée afin de créer un Modèle Numérique de Terrain de 0,3 $\mathrm{m}$ de résolution qui a servi à normaliser le nuage de points $3 \mathrm{D}$ (CGAL, 2013). Plus de détails sur cette approche ainsi que sur les données lidar ainsi que sur celles de l'inventaire terrain sont disponibles dans (Ferraz et al., 2012; Gonçalves et Pereira, 2012). 


\subsection{Estimation de biomasse à partir données d'in- ventaire terrain}

La biomasse par strate est estimée à différents niveaux de détails selon leur nature ou selon les mesures terrain disponibles (Tableau 1). La biomasse stockée dans la canopée adulte est calculée en sommant les estimations faites individuellement par arbre (Equations 15), alors que les biomasses pour la canopée juvénile, le sous-bois et la végétation arbustive sont estimées à l'échelle de la parcelle (Equation 6).

En ce qui concerne les arbres individuels, les équations allométriques ont été obtenues par échantillonnage destructif de 441 eucalyptus (Eucalyptus globulus Labill.) répartis sur 113 parcelles forestières au Portugal (António et al., 2007). De telles équations dépendent à la fois des métriques des arbres individuels $(d b h$, th et $c l=t h-c b h)$, et de coefficients spécifiques à ces parcelles $(a w, a b, a l$, et $a b r)$. Ces dernières sont fonction de la hauteur dominante, qui est définie comme la hauteur moyenne des trois plus grands arbres au sein de la placette (Tableau 1). La biomasse par strate est calculée à partir de la hauteur moyenne $(\mathrm{m})$, du taux de couverture verticale $(\%)$, de la surface $\left(\mathrm{m}^{2}\right)$, et de la densité moyenne apparente $\left(\mathrm{kg} . \mathrm{m}^{-3}\right)$, notées respectivement $\bar{h}, v c c, a$ et $\overline{b d}$ dans le tableau $1 . \overline{b d}$ est la moyenne pondérée des densités apparentes par espèce existante dans les placettes : la moyenne de chaque espèce correspond à sa prédominance (\%, Section 2.1.1). La densité apparente, définie comme la biomasse par volume unitaire, dépend en effet de l'espèce. Les densités apparentes référence pour un grand nombre de plantes existantes au Portugal sont fournies dans (Simões, 2006). Dans notre cas, les moyennes $\overline{b d}$ pour les 40 placettes forestières valent $1,8,1,7$ and $2,5 \mathrm{~kg} \cdot \mathrm{m}^{-3}$ pour respectivement la canopée juvénile, le sous-bois et la végétation arbustive. Cela signifie que les espèces composant la végétation arbustive ont des densités apparentes supérieures à celles composant à la fois la canopée juvénile et le sous-bois.

\subsection{Estimation de biomasse par lidar aéroporté}

Nous décrivons brièvement deux méthodes qui ont été mises au point dans (Ferraz et al., 2012) et (Ferraz et al., soumis) pour l'extraction de variables forestières à partir de nuages de points 3D issus de systèmes lidar aéroportés (Section 2.3.1). Tandis que la première méthode fournit des métriques sur la composante verticale (e.g., densité d'arbres, $t h$ et $c l$ ), la seconde est dédiée à l'obtention de $v c c$ pour chaque strate. Finalement, ces variables forestières sont utilisées comme entrées des équations allométriques détaillés dans le Tableau 1 (Section 2.3.2).

\subsubsection{Extraction de métriques forestières}

Nous utilisons ici l'approche proposée dans (Ferraz et al., 2012), appelée Mean-Shift Adaptatif (AMS 3D). II s'agit d'une méthode de segmentation 3D qui n'utilise comme information pour chaque point que ses coordonnées tri-dimensionnelles (Figure 1a). Chacun de ces segments 3D correspond à un élément unique de végétation, e.g., buisson ou couronne d'arbre, et est assigné à une strate particulière : végétation arbustive, sous-bois ou canopée (Figure 1). Cette méthode de segmentation forestière a été validée sur 40 parcelles, décrites dans la partie 2.1.1. Pour la canopée adulte, la méthode AMS 3D peut détecter correctement 438 arbres sur 649 (67,5\%). Le taux d'arbres correctement détectés (CD) dépend en très grande partie du statut social de sa couronne : il vaut respectivement $98,6 \%, 85,2 \%, 61,4 \%$ et $12,8 \%$ pour les eucalyptus dominants, co-dominants, dominés et opprimés.

Les modèles de régression linéaire expliquent bien mieux la variabilité associée avec th $\left(R^{2}=0,96 \%\right.$, Erreur Quadratique Moyenne - EMQ=0,86 m) qu'avec $c b h$ $\left(R^{2}=0,69 \%, E M Q=2,48 \mathrm{~m}\right)$ pour les arbres correctement détectés. A l'inverse, 9,2\% des arbres détectés avec la méthode AMS 3D correspondent à des faux positifs c'est-à-dire des arbres fictifs, que l'on appellera ensuite incorrectement détectés (ID). Cela veut dire que la méthode AMS 3D a extrait 60 arbres qui n'existent pas sur sur le terrain : cela provient soit à un légère sursegmentation (une couronne sera divisée en deux segments) soit à des effets de bord (des arbres dont le tronc est en dehors des limites de la parcelle peuvent avoir une partie de leur couronne à l'intérieur de la parcelle).

Les hauteurs moyennes $\bar{h}$ de la canopée juvénile, du sous-bois et de la végétation arbustive ont été validées à l'échelle de la placette. La corrélation entre mesures terrain et celles dérivées des données lidar a également été estimée par régression linéaire. Les artefacts, qui représentent respectivement $20 \%, 16,7 \%$ et $7,3 \%$ des parcelles pour la canopée juvénile, le sous-bois et la végétation arbustive, ont été éliminés des régressions linéaires. Les modèles linéaires avec des EMQ satisfaisants $(0,31 \mathrm{~m}, 0,96 \mathrm{~m}$ et $0,15 \mathrm{~m})$ donnent respectivement des valeurs de $R^{2}$ de $0,92,0,62$ et 0,7 .

Le taux de couverture verticale $(v c c)$ est elle extraite grâce à la méthode présentée dans (Ferraz et al., soumis). Le $v c c$ a été calculé pour chaque strate en utilisant là aussi directement les nuages de points 3D correspondant soit à la canopée juvénile, soit au sous-bois, soit à la végétation arbustive, selon la méthode AMS 3D (Figure 1b). Une méthode statistique non-paramétrique appelée Kernel Density Estimator (KDE, (Parzen, 1962)) est utilisée pour convertir les mesures discrètes (i.e., les échos lidar) en des surfaces continues lisses représentant des densités spatiales que l'on appellera par analogie des modèles de densité de probabilité de canopée (Canopy Probability Density Models - CPDM - Figures $1 c$ et $d$ ). Le CPDM est ainsi une carte de probabilité de présence de végétation pour l'intégralité de la parcelle : les plus fortes probabilités sont assignées aux zones de plus fortes densités de points. Les cartes de vcc peuvent être obtenues à partir des CPDM en seuillant la densité de probabilité en dessous de laquelle on 


\begin{tabular}{|c|c|c|c|}
\hline \multicolumn{3}{|c|}{ Biomasse (kg) } & \multicolumn{3}{|c|}{} \\
\hline \multirow{4}{*}{ Arbres } & Tronc & $w_{w}=0,0104 \times d b h^{a w} \times t h^{1,35942}$ & $(1)$ \\
\cline { 2 - 4 } & Ecorce & $w_{b}=0,00061 \times d b h^{a b} \times t h^{1,08249}$ & $(2)$ \\
\cline { 2 - 4 } & Feuilles & $w_{l}=0,04579 \times d b h^{a l} \times c l^{0,82504}$ & $(3)$ \\
\cline { 2 - 4 } & Branches & $w_{b r}=0,02487 \times d b h^{a b r} \times c l^{0,02478}$ & $(4)$ \\
\cline { 2 - 4 } & Total & $w_{t}=w_{w}+w_{b}+w_{l}+w_{b r}$ & $(5)$ \\
\hline \multicolumn{2}{|c|}{ Strate } & $w_{\text {strate }}=\bar{h} \times v c c \times a \times \bar{b} d$ & $(6)$ \\
\hline \multicolumn{2}{|c|}{$\boldsymbol{d b h}(\mathbf{c m})$} & $d b h=\frac{0,6073 \times t h}{1-0,0116 \times t h}$ & $(7)$ \\
\hline \multicolumn{2}{|c|}{ Arbres } & \multicolumn{3}{|c}{}
\end{tabular}

TABLEAU 1 : Equations pour l'estimation de la biomasse aérienne et du diamètre à hauteur de poitrine $(d b h)$.
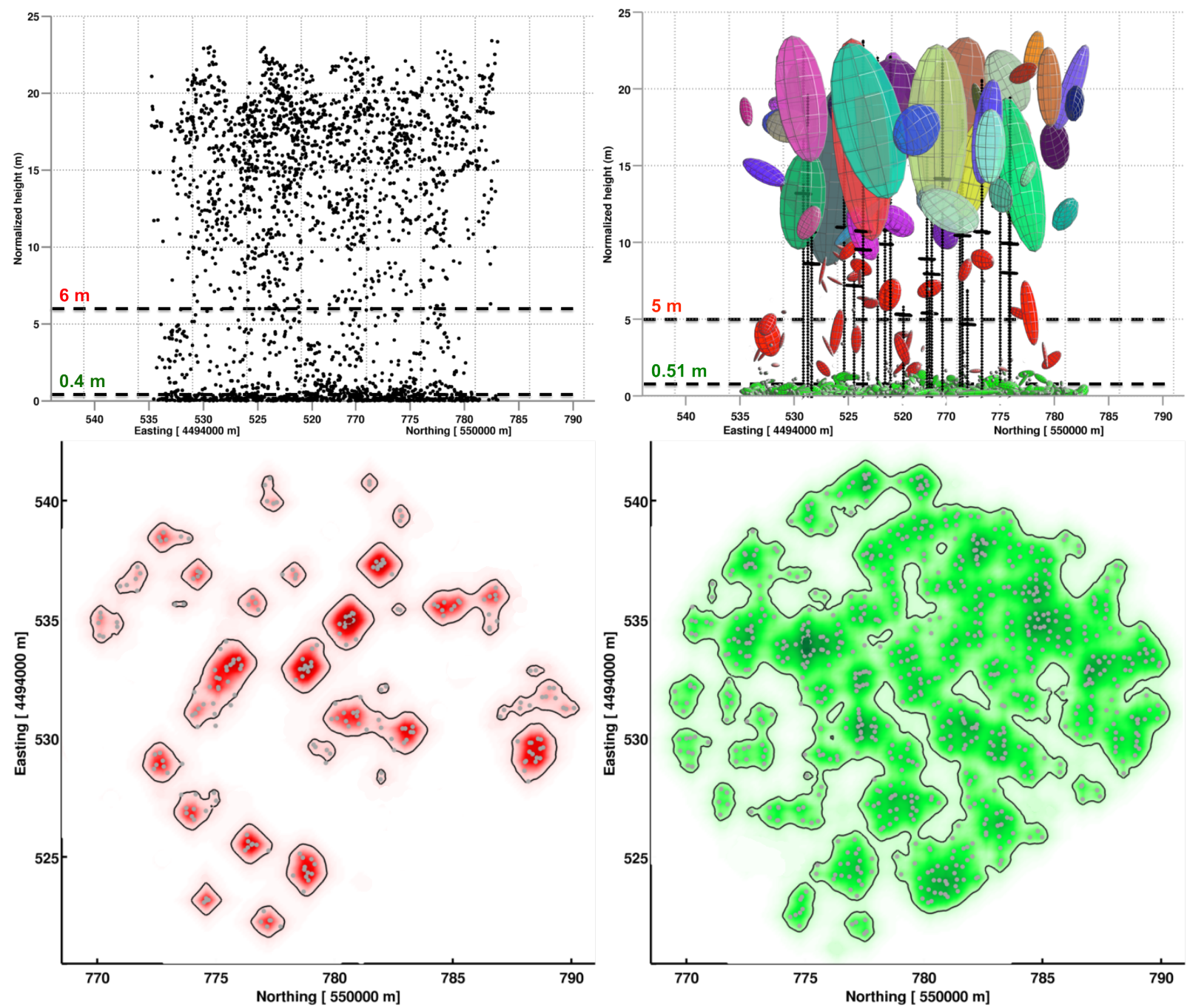

FIGURE 1 : (a) Nuage de points 3D brut sur une parcelle adulte, avec les hauteurs moyennes de sous-bois (rouge) et de végétation arbustive (vert), mesurées sur le terrain. Celles-ci sont représentées par des lignes horizontales en pointillés. (b) Extraction individuelle d'arbres par la méthode AMS 3D : chaque couronne est représentée par des ellipsoïdes de couleur rouge, verte et multicolores, respectivement pour le sous-bois, la végétation arbustive et la canopée. Les lignes pointillées représentent les hauteurs moyennes estimées pour le sous-bois (rouge) et la végétation arbustive (vert). Les arbres mesurés sur le terrain sont représentés par des segments verticaux noirs. (c) et (d) représentent les modèles de densité de probabilité de canopée pour le sous-bois (rouge) et la végétation arbustive (vert). Les points lidar utilisés pour les calculer sont indiqués en gris. Les valeurs de $v c c$ correspondantes valent $23,7 \%$ and $74,6 \%$, alors qu'elles ont été estimées respectivement sur le terrain à $32 \%$ et $80 \%$. 
pense qu'il n'y a pas de végétation (contours noirs sur les figures $1 c$ et $1 \mathrm{~d}$ ). Le rapport entre les zones couvertes par la végétation et celle de la placette forestière donne la valeur de $v c c$. Dans ce cas, les artefacts correspondent à 20\%,13,3\% and 10\% des parcelles pour respectivement la canopée juvénile, le sous-bois et la végétation arbustive. Les modèle slinéaires expliquent 0,85 , 0,85 et 0,6 de la variabilité associée aux $v c c$ respectifs. Les EMQ respectifs valent eux $8,5 \%, 6,4 \%$ et $20,8 \%$.

\subsubsection{Biomasse par strate}

La biomasse par strate est estimée en utilisant les équations allométriques présentées dans le tableau 1. Pour la canopée adulte, la biomasse est obtenue de manière individuelle pour chaque arbre en appliquant les équations 1 à 5 . En pratique, nous profitons du fait que seuls des eucalyptus peuplent la canopée. Autrement, il aurait été nécessaire de d'abord discriminer les différentes espèces pour sélectionner les équations allométriques adéquates. La méthode AMS 3D fournit toutes les variables inconnues sauf le $d b h$. Cela est en effet incompatible avec les densités de points lidar employées pour des applications forestières grande échelle (i.e., entre 4 et $20 \mathrm{pts} / \mathrm{m}^{2}$, (Jakubowski et al., 2013)). En conséquence, le $d b h$ est généralement dérivé des mesures de th via des équations allométriques spécifiques (Popescu, 2007). Dans notre étude, nous appliquons l'équation 7 établie par Soares et Tomé (2010), en utilisant les mesures terrain décrites dans la Section 2.1.1.

Les biomasses pour la canopée juvénile, le sous-bois et la végétation arbustive sont estimées strate par strate en appliquant l'équation 6. Alors que la méthode AMS 3D fournit $\bar{h}$ pour chaque strate, son $v c c$ est calculé à partir du CPDM correspondant. Néanmoins, il n'y a pas de méthodologie pour obtenir de manière directe $\overline{b d}$ pour les strates sous-jacentes. Son estimation à partir de données lidar aéroporté reste une problématique de recherche majeure, qui nécessite plus d'investigation, comme discuté dans la partie 3. Dans cet article, nous utilisons une valeur de $\overline{b d}$ spécifique à chaque strate et unique pour l'intégralité des zones d'étude. Tout d'abord, les espèces qui composent chaque strate ont été identifiées pendant l'inventaire terrain (Section 2.1.2). Les densités apparentes de référence correspondantes fournies dans (Simões, 2006) ont ensuite été assignées à chaque espèce. La moyenne des densités apparentes pour chaque strate a été calculée afin d'estimer finalement les $\bar{b} d$ correspondants : 1,76, 2,21 and 2,46 kg.m ${ }^{-3}$ pour la canopée juvénile, le sous-bois et la végétation arbustive. II faut noter que, contrairement à son estimation sur le terrain (Section 2.2), dans ce cas présent, le $\overline{b d}$ par strate ne varie pas en fonction de la parcelle forestière puisque la prédominance des types de plantes (\%) n'est pas prise en compte.

\section{Résultats et discussions}

Tout d'abord, nous avons analysé les résultats strate par strate : canopée adulte, canopée juvénile, végétation arbustive et sous-bois (Section 3.1). La biomasse aérienne (agb) à l'échelle de la parcelle forestière est ensuite obtenue par l'addition des contributions individuelles estimées de chaque des strates (Section 3.2).

\subsection{Biomasse par strate}

Selon la biomasse estimée à partir des données terrain, $7,9,7,7,6,1$ et $78,3 \%$ de la biomasse totale est stockée dans la végétation arbustive, le sous-bois, les canopées juvénile et adulte. En moyenne, elles comprennent $6,7,8,9,29,3$ et 93,7 mégagrammes $(\mathrm{Mg})$, avec des écart-types respectifs de 4,6, 6,8, 10 et 66,1 Mg. Une régression linéaire a été employée pour étudier la corrélation entre les biomasses estimées par placette à partir des données terrain et par analyse de données lidar. Alors que l'erreur absolue est estimée via l'erreur quadratique moyenne (EMQ), cette variable ne peut pas être utilisée pour comparer de manière robuste les erreurs entre strates. Devant la grande variabilité de biomasse stockée dans les différentes strates, à savoir la canopée comparée aux autre strates, nous avons homogénéisé l'EMQ en moyennant la biomasse obtenue à partir des données terrain, i.e., $E M Q / \overline{a g b}$. $\overline{a g b}$ est la valeur moyenne des biomasses estimées sur le terrain pour les 40 placettes. Cela permet de contrôler les différences en amplitude entre les différentes strates (Zolkos et al., 2013).

L'estimation à l'échelle de la parcelle pour la canopée adulte, calculée comme la somme des biomasses par arbre, est extrêmement corrélée $\left(R^{2}=0,99\right)$ avec les valeurs obtenues à partir des données terrain (Figure 2a et Tableau 1). Cependant, il faut prendre ce résultat avec précaution. Le diamètre à hauteur de poitrine $(d b h)$ des arbres correctement détectés (CD) a été calculé à partir de l'équation 7 , en fonction de l'estimation de $t h$ issue des données lidar $\left(R^{2}=0,78, E M Q=2,1 \mathrm{~cm}\right)$. Ensuite, la biomasse des arbres $C D$ a été calculée en utilisant les équations 1-5 $\left(R^{2}=0,81, E M Q=22,63 \mathrm{~kg}\right)$. Par conséquent, la biomasse dérivée des mesures lidar est plus corrélée à celle estimée à l'échelle de la parcelle qu'à celle obtenue à l'échelle de l'arbre. En fait, la biomasse à l'échelle de la parcelle est obtenue en sommant à la fois les biomasses des arbres correctement et incorrectement détectés. Cela signifie que les arbres ID, i.e., les faux positifs générés par la méthode AMS 3D, compensent en partie la biomasse des arbres non détectés (UD). Néanmoins, cela n'explique pas la corrélation forte à l'échelle de la parcelle puisque le pourcentage d'arbres UD $(32,5 \%)$ est bien supérieur à celui des arbres ID $(9,2 \%)$. Ce résultat montre avant tout que l'approche fondée sur les données lidar est capable de détecter les arbres qui contiennent le plus de biomasse au sein des parcelles. En effet, dans notre zone d'étude, beaucoup d'arbres UD correspondent à des arbres opprimés, qui 
stockent peu de biomasse et qui ne sont pas des contributeurs significatifs pour l'estimation à l'échelle de la placette.

Les résultats obtenus pour la canopée juvénile, le sous-bois et la végétation arbustive dépendent fortement de la strate de la forêt. La corrélation entre les estimations terrain et lidar est modérée pour la canopée juvénile et le sous-bois (respectivement, $R^{2}=0,38$ et $R^{2}=0,37$ ), alors qu'elle est bien plus élevée pour la végétation arbustive $\left(R^{2}=0,65\right)$. En ce qui concerne l'erreur quadratique moyenne (\%), les résultats sont satisfaisants pour la canopée juvénile et la végétation arbustive (respectivement 56,7 et $53,3 \%$ ) mais décevants pour le sousbois $(101,3 \%)$. Plusieurs facteurs expliquent de tels résultats. Tout d'abord, l'inventaire terrain a été effectué à l'échelle de la parcelle et est donc beaucoup moins fiable que pour la canopée adulte où chaque arbre a été individuellement mesuré. Ensuite, les méthodes mises au point pour l'analyse de zones forestières par lidar aéroporté (AMS 3D et CPDM) introduisent des erreurs sur l'estimation de la biomasse car elles fournissent des valeurs de $\bar{h}$ et de $v c c$ avec un certain degré d'incertitude et génèrent des artefacts (Section 2.3.1).

Les résultats dépendent également de la capacité du lidar à caractériser géométriquement la structure de la forêt. On sait très bien que la végétation haute masque en partie la végétation sous-jacente. Ainsi, ces strates peuvent être sous-échantillonnées dans le nuage de points 3D en comparaison avec la canopée. Malgré cela, l'approche lidar trouve une corrélation plus forte pour la végétation arbustive que pour la canopée juvénile et le sous-bois. II est probable que cela soit dû aux espèces qui composent la végétation arbustive : elles ont des densités apparentes supérieures (i.e., plus de biomasse par volume unitaire) aux espèces qui peuplent la canopée juvénile et le sous-bois. En utilisant les mesures fournies par l'inventaire terrain et les densités apparentes référence (Simões, 2006), les valeurs moyennes de $\overline{b d}$ pour les 40 parcelles de notre zone d'étude valent $1,8,1,7,2,5 \mathrm{~kg} \cdot \mathrm{m}^{-3}$ pour, respectivement la canopée juvénile, le sous-bois et la végétation arbustive. Ainsi, les plus fortes densités apparentes indiquent des plantes plus denses, et de telles plantes ont une plus forte probabilité de générer un écho lidar que les plantes peu denses. En conclusion, les résultats indiquent que de meilleures estimations sont obtenues pour la végétation arbustive car c'est une strate plus dense composée de plantes avec des densités apparentes supérieures.

L'effet d'occlusion causée par la canopée semble avoir peu d'impact puisque les résultats les moins satisfaisants ont été obtenus pour la canopée juvénile et le sous-bois, qui correspondent à des strates hautes.

Finalement, il faut noter que le paramétrage de $\overline{b d}$ a un impact non négligeable sur les résultats (Equation 6). Le calcul de $\overline{b d}$ nécessite de connaître quelles espèces composent les parcelles forestières ainsi que leurs dominances (Section 2.1.1).

Néanmoins, l'identification d'espèces à partir de don- nées lidar n'a été abordée dans la littérature que pour la couche supérieure de la végétation, et ceci avec une efficacité limitée. En fait, certaines contraintes limitent le développement d'une telle technique. Comparativement à la canopée, les broussailles, buissons et formations herbacées sont généralement plus riches en biodiversité, ce qui accroît fortement la complexité de l'approche. De plus, devant l'incapacité des méthodes fondées sur les données lidar à discriminer les espèces pour des peuplements mélangés, les chercheurs ont traité ce problème en tirant avantage de la synergie entre lidar aéroporté et imagerie optique hyperspectrale (Dalponte et al., 2012). En effet, la végétation sous la canopée n'est pas visible avec de l'imagerie optique géospatiale et, en conséquence, nous pensons que l'estimation de $\overline{b d}$ à partir de données auxiliaires est une solution viable pour ces strates. Nous suggérons d'obtenir des valeurs moyennes bien adaptées à une zone de forêt ou éco-région spécifique. Les espèces végétales attendues dans un tel écosystème peuvent être retrouvées soit avec des connaissances a priori sur la forêt, soit à partir d'études écologiques : cartographie de la biodiversité ou distribution biogéographique des espèces végétales (Gachet et al., 2005 ; Hazen, 2009).

\subsection{Biomasse aérienne}

Les biomasses dérivées par lidar aéroporté par strate ont été sommées afin de calculer l'agb pour chacune des 40 parcelles forestières. La corrélation entre les agb estimées sur le terrain et avec les données lidar a été examinée par régression linéaire (Figure $2 b$ et Tableau 2). On note que celle-ci est très forte puisque $\mathrm{R}^{2}=0,99$, de manière similaire à la relation obtenue pour la canopée seule. Ce résultat était attendu puisque, dans notre zone d'étude, la plus grande partie de la biomasse forestière se trouve dans la canopée adulte. Cependant, l'erreur absolue (EMQ) a été améliorée jusqu'à 1,7 Mg.ha ${ }^{-1}$. De manière plus significative, on note une grande amélioration des résultats en terme de EMQ (en $\%$ : $18,1 \%$ et $0,4 \%$ pour respectivement les biomasses de la canopée adulte seule et de la végétation d'une placette dans son ensemble.Nos résultats indiquent que l'incertitude dans l'estimation de l'agb peut être fortement réduite si une stratification est menée et l'estimation faite à l'échelle de la strate.

Bien que les résultats peuvent varier selon ces dernières, notre approche estime tout de même la biomasse aérienne de manière satisfaisante. Ils permettent de rendre compte de la variabilité de l'agb pour des structures forestières complexes et très stratifiées, même si les résultats sont moins bons pour le sous-bois. Au final, il faut noter que notre approche n'a été validée que pour une petit nombre de placettes forestières et que l'on attend un erreur moindre en augmentant ce nombre (Zolkos et al., 2013). 

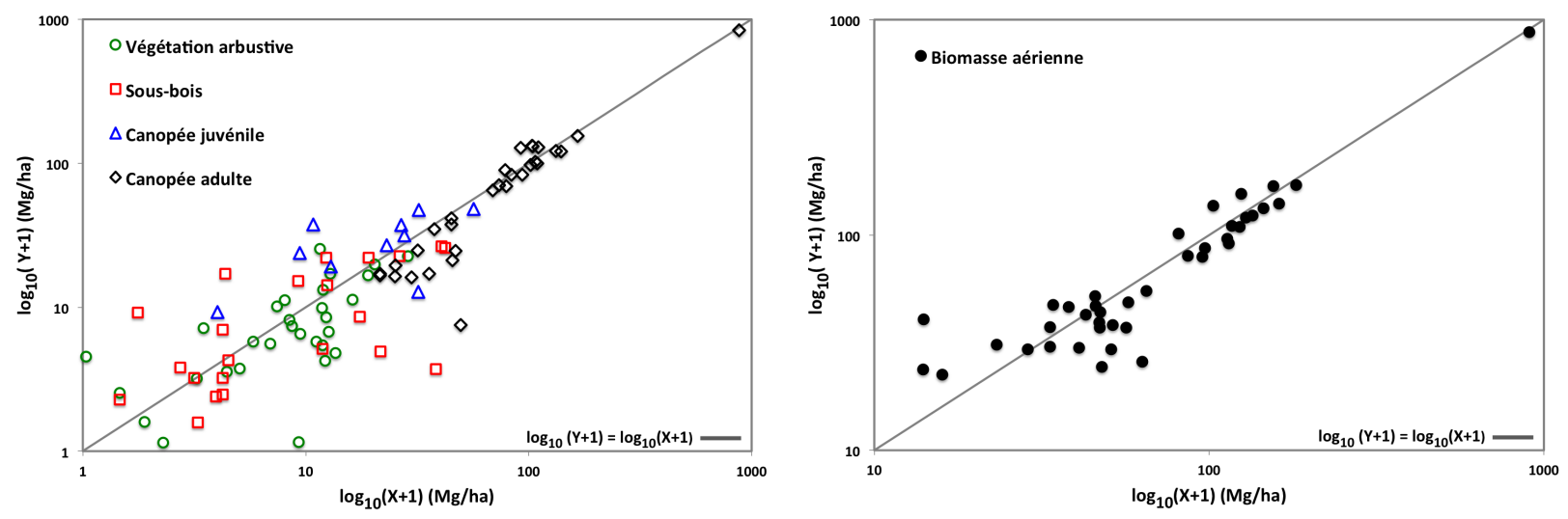

FIGURE 2 : Biomasse estimée à partir des données terrain (axe $x$ ) comparée à celle dérivée à partir des données lidar (axe $y$ ) pour chaque strate (a) et la biomasse aérienne (b).

\begin{tabular}{|c|c|c|c|c|c|}
\hline Strate de forêt & $\mathrm{n}$ & $\mathrm{R}^{2}$ & $\mathrm{EMQ}$ & $\mathrm{EMQ}(\%)$ & $\Delta\left(\mathrm{Mg}^{\mathrm{h}} \mathrm{ha}^{-1}\right)$ \\
\hline Canopée adulte & 30 & 0,99 & 18 & 18,1 & $-5,8$ \\
\hline Canopée juvénile & 10 & 0,38 & 13,3 & 56,7 & $+5,8$ \\
\hline Sous-bois & 30 & 0,37 & 9,9 & 101,3 & $-0,8$ \\
\hline Végétation arbustive & 40 & 0,65 & 4,1 & 53,3 & -0.7 \\
\hline \hline Biomasse aérienne & 40 & 0,99 & 16,3 & 0,4 & $-4,4$ \\
\hline
\end{tabular}

TABLEAU 2 : Paramètres de la régression linéaire entre biomasse estimée par strate par les données terrain contre les données lidar. $n$ correspond au nombre de placettes et des valeurs négatives de $\Delta$ à une sous-estimation de la biomasse.

\section{Conclusion}

Dans cet article, nous avons démontré la pertinence du lidar aéroporté pour l'estimation de biomasse à une échelle équivalente à celle des inventaires terrain couramment pratiqués dans les inventaires forestiers portugais (i.e., à l'échelle de l'arbre pour la canopée et à celle de la parcelle pour le sous-bois et la végétation arbustive). Même si la précision des résultats dépend fortement de la strate d'intérêt, la méthode fondée sur les données lidar prédit de de manière fiable la biomasse aérienne totale de notre zone d'étude car celles-ci sont capables de bien décrire la structure géométrique des strates de végétation qui contiennent la quantité la plus important de la biomasse. Les études précédentes menées à partir de données de télédétection ont conclu que les erreurs attendues quant à l'estimation de la biomasse sont de l'ordre de $20{\mathrm{Mg} . \mathrm{ha}^{-1}}^{-1}$ ou $20 \%$ des estimations faites sur le terrain (Houghton et al,. 2009; Hall et al., 2011). Notre approche est elle capable d'obtenir une biomasse aérienne avec une erreur quadratique moyenne de $0,4 \%$. Cela montre qu'elle est une méthode fiable pour obtenir une référence robuste pour son extrapolation sur de larges étendues par analyse d'images satellites. Alors que l'inventaire terrain effectué pour notre étude fournit des valeurs de biomasse aérienne pour environ $8000 \mathrm{~m}^{2}$ (qui correspond aux 40 parcelles forestières), il faut noter que les données lidar couvrent près de $7 \mathrm{~km}^{2}$ de forêt d'eucalyptus. Ainsi, elles évaluent la variabilité spatiale de la biomasse sur de plus vastes zones.

En effet, la disponibilité à des points-clé du globe (notamment des zones forestières où aucune mesure terrain n'existe, comme sur des vastes zones de forêts tropicales) et à plusieurs dates, d'estimations précises de biomasse aérienne par lidar aéroporté pourrait permettre de satisfaire aux besoins du programme REDD des Nations Unies en un temps acceptable. A notre avis, la technique de télédétection par lidar aéroporté va jouer un rôle crucial comme appui aux politiques de régulation du changement climatique. Elle a besoin pour cela d'être intégrée dans un cadre multi-résolution plus global, couplée avec des techniques de télédétection satellitaire pour une estimation mondiale de la biomasse aérienne. Pour cela, plus d'efforts doivent être faits dans le développement et la validation de méthodes robustes d'extraction d'arbres individuels, applicables à différents écosystèmes forestiers. En fait, nous ne croyons pas qu'il existe une raison technologique pour laquelle une campagne lidar bien planifiée suivie d'une chaîne de traitements de nuages de points $3 \mathrm{D}$ adéquate ne serait pas capable de restituer les éléments les plus significatifs en terme de biomasse pour n'importe quel type de forêt. Pour des paysages extrêmement complexes, il reste évident que des approches plus sophistiquées doivent être développées. Par exemples, des forêts mixtes ne peuvent être correctement analysées sans profiter de la synergie des données lidar avec des images hyper- 
spectrales afin de discriminer les espèces d'arbres et sélectionner l'équation allométrique de biomasse la plus adéquate (Asner et al., 2008 ; Féret et Asner, 2013).

La principale limitation de notre méthode à l'heure actuelle est que l'utilisation seule des données lidar aéroporté ne permet que d'acquérir des zones relativement petites. Cela est autant dû aux coûts qu'aux aspects logistiques en terme de stockage et de gestion de très grands volumes de données.

\section{Remerciements}

Ce travail a bénéficié du financement de la Fondation Portugaise pour la Science et la Technologie (contrat PEst-OE/ EEI/UI308/2014) ainsi que de l'Agence Nationale de la Recherche dans le cadre du projet FORESEE (ANR-2010-BIOE-008).

\section{Références}

AFN, 2009. Instruçoes para o trabalho de campo do Inventario Florestal Nacional. In, IFN 2005/2009 (p. 62). Lisboa, Portugal : Divisao para a Intervençao Florestal, Autoridade Florestal Nacional, Direcçao de Unidade de Gestao Florestal, Divisao para a Intervençao Florestal. António, N., Tomé, M., Tomé, J., Soares, P., and Fontes, L., 2007. Effect of tree, stand, and site variables on the allometry of Eucalyptus globulus tree biomass. Canadian Journal of Forest Research 37(5):895-906. Asner, G.P., Knapp, D.E., Kennedy-Bowdoin, T., Jones, M.O., Martin, R.E., Boardman, J., and Hughes, R.F., 2008. Invasive species detection in Hawaiian rainforests using airborne imaging spectroscopy and LiDAR. Remote Sensing of Environment 112(5):1942-1955.

CGAL, Computational Geometry Algorithms Library, http://www.cgal.org, accessed October 18, 2013.

Dalponte, M., Bruzzone, L., and Gianelle, D., 2012. Tree species classification in the Southern Alps based on the fusion of very high geometrical resolution multispectral/hyperspectral images and LiDAR data. Remote Sensing of Environment 123:258-270.

Féret, J.B. and Asner, G.P., 2013. Tree Species Discrimination in Tropical Forests Using Airborne Imaging Spectroscopy. IEEE Transactions on Geoscience and Remote Sensing 51(1): 73-84.

Ferraz, A., Bretar, F., Jacquemoud, S., Gonçalves, G., Pereira, L., Tomé, M., and Soares, P., 2012. 3-D mapping of a multi-layered Mediterranean forest using ALS data. Remote Sensing of Environment 121:210-223.

Ferraz, A., Mallet, C., Jacquemoud, S., Gonçalves, G., Soares, P., Pereira, L., Tomé, M., and Bretar, F., (submitted). Canopy probability density model : a new ALS-derived product to derive single forest stratum cover. IEEE Transactions on Geoscience and Remote Sensing.

Gachet, S., Vela, E., and Tatoni, T., 2005. BASECO : a floristic and ecological database of Mediterranean
French flora. Biodiversity and Conservation 14(4):10231034.

Gobakken, T., Næsset, E., Nelson, R., Bollandsås, O.M., Gregoire, T.G., Ståhl, G., Holm, S., Ørka, H.O., and Astrup, R., 2012. Estimating biomass in Hedmark County, Norway using national forest inventory field plots and airborne laser scanning. Remote Sensing of Environment 123:443-456.

Gonçalves, G., and Pereira, L., 2012. A thorough accuracy estimation of DTM produced from airborne full-waveform laser scanning data of unmanaged Eucalyptus plantations. IEEE Transactions on Geoscience and Remote Sensing 50(8):3256-3266.

Gonzalez, P., Asner, G.P., Battles, J.J., Lefsky, M., Waring, K.M., Palace, M., 2010. Forest carbon densities and uncertainties from Lidar, QuickBird, and field measurements in California. Remote Sensing of Environment 114:1561-1575.

Hall, F.G., Bergen, K., Blair, J.B., Dubayah, R., Houghton, R., Hurtt, G., Kellndorfer, J., Lefsky, M., Ranson, J., Saatchi, S., Shugart, H.H., and Wickland, D., 2011. Characterizing 3D vegetation structure from space : Mission requirements. Remote Sensing of Environment 115:2753-2775.

Hazen, H., 2009. Biodiversity Mapping. In K. Editorsin-Chief : Rob, and T. Nigel (Eds.), International Encyclopedia of Human Geography (pp. 314-319). Oxford : Elsevier.

Houghton, R.A., Hall, F., and Goetz, S., 2009. Importance of biomass in the global carbon cycle. Journal Geophysics Research 114(G2).

Houghton, R.A., Hall, F., and Goetz, S.J., 2010. The role of science in REDD. Carbon Management 1:253-259.

Hu, B., Li, J., Jing, L., and Judah, A., 2014. Improving the efficiency and accuracy of individual tree crown delineation from high-density LiDAR data. International Journal of Applied Earth Observation and Geoinformation 26:145-155.

Hug, C., Ullrich, A., Grimm, A., 2004. Litemapper-5600 - A Waveform-Digitizing LIDAR Terrain and Vegetation Mapping System. International Archives of Photogrammetry, Remote Sensing and Spatial Information Sciences 36 (Part 8/W2), 24-29.

Hyyppä, J., Hyyppä, H., Leckie, D., Gougeon, F., Yu, X., and Maltamo, M., 2008. Review of methods of small-footprint airborne laser scanning for extracting forest inventory data in boreal forests. International Journal of Remote Sensing 29(5):1339-1366.

Jakubowski, M.K., Guo, Q., Kelly, M., 2013. Tradeoffs between lidar pulse density and forest measurement accuracy. Remote Sensing of Environment 130:245-253. Korhonen, L., Korpela, I., Heiskanen, J., and Maltamo, M., 2004. Airborne discrete-return LIDAR data in the estimation of vertical canopy cover, angular canopy closure and leaf area index. Remote Sensing of Environment 115:1065-1080.

Morsdorf, F., Meier, E., Kötz, B., Itten, K.I., Dobbertin, M., and Allgöwer, B., 2004. LIDAR-based geometric 
reconstruction of boreal type forest stands at single tree level for forest and wildland fire management. Remote Sensing of Environment 92:353-362.

Parzen, E., 1962. On estimation of a probability density function and mode. The Annals of Mathematical Statistics 33:1065-1076.

Popescu, S., 2007. Estimating biomass of individual pine trees using airborne lidar. Biomass and Bioenergy 31:646-655.

Popescu, S., and Wynne, R., 2004. Seeing the trees in the forest : using Lidar and multispectral data fusion with local filtering and variable window size for estimating tree height. Photogrammetric Engineering \& Remote Sensing 70(5):589-604.

Reitberger, J., Schnörr, C., Krzystek, P., and Stilla, U., 2009. 3D segmentation of single trees exploiting full waveform LIDAR data. ISPRS Journal of Photogrammetry and Remote Sensing 64(6):561-574.

Saatchi, S., Marlier, M., Chazdon, R., Clark, D., and Russell, A., 2011. Impact of spatial variability of tropical forest structure on radar estimation of aboveground biomass. Remote Sensing of Environment 115:28362849.

Simões, S., 2006. Expansão ao Alentejo e Algarve de uma curva de acumulação pós-fogo para a biomassa arbustiva. In. Lisboa : Universidade Técnica de Lisboa, Instituto Superior de Agronomia, Portugal.

Soares, P., and Tomé, M., 2010. Airborne laser scanning technologies? need to estimate tree variables normallyobtained in traditional forest inventory. In : IUFRO conference on mixed and pure forest in a changing world, Vila Real, Portugal.

Solberg, S., Næsset, E., and Bollandsas, O., 2006. Single tree segmentation using airborne laser scanner data in a structurally heterogeneous spruce forest. Photogrammetric Engineering \& Remote Sensing 72:13691378.

Stokes, J., Ashmore, C., Rawlins, L., and Sirois, L., 1989. Glossary of Terms Used in Timber Harvesting and Forest Engineering. In U. General Technical Report SO-73, Forest Service, Southern Forest Experiment Station (Ed.) (p. 33). New Orleans, USA.

Wing, B.M., Ritchie, M.W., Boston, K., Cohen, W.B., Gitelman, A., and Olsen, M.J., 2012. Prediction of understory vegetation cover with airborne lidar in an interior ponderosa pine forest. Remote Sensing of Environment 124:730-741.

Wulder, M.A., White, J.C., Nelson, R.F., Næsset, E., Ørka, H.O., Coops, N.C., Hilker, T., Bater, C.W., and Gobakken, T., 2012. Lidar sampling for large-area forest characterization : A review. Remote Sensing of Environment 121:196-209.

Zolkos, S.G., Goetz, S.J., and Dubayah, R., 2013. A meta-analysis of terrestrial aboveground biomass estimation using lidar remote sensing. Remote Sensing of Environment 128:289-298. 